\title{
Usefulness of WBC Count to Mean Platelet Volume Ratio in Predicting Short-Term (30 Days) Major Adverse Cardiac Events in Patients Presenting with Acute Coronary Syndrome and its Comparison in Males and Females
}

\author{
Sheshidhar Madaka' \\ ${ }^{1}$ Department of Cardiology, NIMS Hyderabad, India628897
}

Ind J Car Dis Wom 2021;6:88-97.

\begin{abstract}
Address for correspondence Dr. Sheshidhar Madaka, MD, DM Resident, Department of Cardiology, NIMS Hyderabad, India (e-mail: sheshidharmadaka@gmail.com).
\end{abstract}

\begin{abstract}
Keywords

- White blood cell (WBC) to mean platelet volume (MPV) ratio (WMR)

- major adverse cardiac events (MACE)

- acute coronary syndrome (ACS)

- males and females

Background: Among cardiovascular illnesses, acute coronary syndrome (ACS) events are associated with higher mortality and morbidity. The mechanism of inflammation involved in the ACS includes atherosclerotic plaque development, followed by development of plaque rupture and thrombosis. The present study is to determine whether white blood cell count to mean platelet volume ratio (WMR) can predict short-term (30 days) major adverse cardiac events (MACE) in ACS patients, and also compare WMR and MACE in males and females.

Aim: To detect usefulness of white blood cell (WBC) count to WMR in predicting short term (30 days) MACE in patients presenting with ACS and compare values and MACE events in males and females.

Material and Methods: The present study was conducted in a tertiary-care hospital from September 2020 to December 2020. A total of 60 patients, who presented with $\mathrm{ACS}$ and were undergoing percutaneous coronary intervention $(\mathrm{PCI})$, fulfilled the selection criterion; hence, a total of 60 patients were selected for the study after taking informed consent. The clearance from the Institutional Ethical committee (IEC) was obtained prior to the initiation of the study. All the necessary investigations were done in patients who fulfilled the inclusion criteria. The results of the study were systematically selected and analysis done statistically. The study included all patients with clinical suspicion of ACS for more than 18 years and excluded non-ACS patients, patients with malignancy history, inflammatory diseases, autoimmune disorders, infections, and those who are immunosuppressed.
\end{abstract}

DOI https://doi.org/ $10.1055 / \mathrm{s}-0041-1732513$
(C) 2021. Women in Cardiology and Related Sciences.

This is an open access article published by Thieme under the terms of the Creative Commons Attribution-NonDerivative-NonCommercial-License, permitting copying and reproduction so long as the original work is given appropriate credit. Contents may not be used for commercial purposes, or adapted, remixed, transformed or built upon. (https://creativecommons.org/licenses/by-nc-nd/4.0/).

Thieme Medical and Scientific Publishers Pvt. Ltd. A-12, 2nd Floor, Sector 2, Noida-201301 UP, India 
Results: In the present study, receiver operating characteristic (ROC) curve showed cutoff value of WMR as 1059 with area under curve (AUC) of 0.6 (95\% Cl 0.4-0.9). MACE occurred in 12 patients and mortality occurred in 6 patients. Among MACE, recurrent myocardial infarction (REMI) occurred in 7 (35\%) cases, arrhythmias occurred in $5(25 \%)$, and cardiogenic shock occurred in $8(40 \%)$ cases.

WMR with a cutoff value of 1059 was significant and highly accurate in predicting MACE, with sensitivity of $91.6 \%$, specificity of $87.5 \%$, PPV of $64.7 \%$, NPV of $97.6 \%$, and diagnostic accuracy of $88.3 \%$. Compared to males, females have less sensitivity to, high specificity to, and high diagnostic accuracy of WMR ratio in predicting MACE. MACE did not show any gender predilection.

Conclusion: In patients, who presented with ACS, high WMR values were associated with worse short-term outcomes and independently predicted short-term MACE. Compared to males, females have less sensitivity to, high specificity to, and high diagnostic accuracy of WMR ratio in predicting MACE.

\section{Abstract Image}

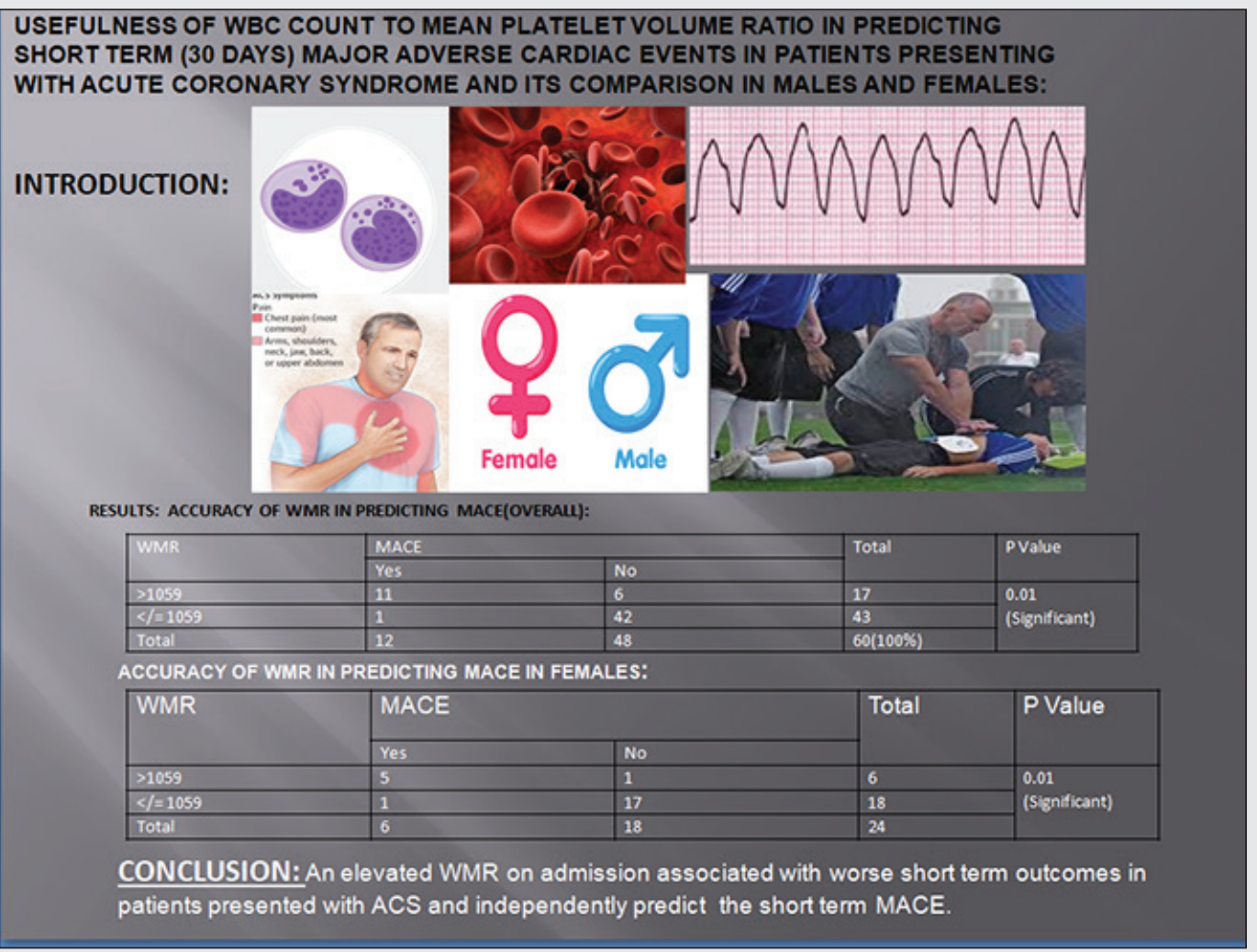

\section{Introduction}

Among cardiovascular illnesses, acute coronary syndrome (ACS) events are associated with higher mortality and morbidity. ${ }^{1}$ Majority of the cardiovascular disease risk burden will be taken by the Indian subcontinent in the future. ${ }^{2}$ The mechanism of inflammation involved in ACS includes atherosclerotic plaque development, followed by development of plaque rupture and thrombosis. ${ }^{3}$
ACS events are not only due to the stenosis of coronary arterial system but also due to other pathophysiological mechanisms involved in the development of acute coronary events. In the development of acute coronary events, leukocytes play an important role through the processes of inflammation. ${ }^{4}$ Macrophages and T-cells, which are located in the atherosclerotic plaque core, get activated when endothelium is injured, and there is release of cytokines and procoagulants by these cells, which promote the thrombus formation. These 
mechanisms increase the risk of thrombogenicity and the development of ACS. ${ }^{5}$

Increased leukocyte (white blood cell [WBC]) count is helpful in predicting clinical outcome in patients with ACS. ${ }^{6}$ Mean platelet volume (MPV) is a marker of platelet activation is an inflammatory marker, which can be useful in assessing prognosis in patients with ACS. ${ }^{7}$ As a combination of both these markers (WBC and MPV), WBC count to MPV ratio (WMR) has been recently found as a novel noninvasive marker for predicting long-term outcomes in patients with $\mathrm{ACS}^{8}$. However, the use of this novel marker for assessing prognosis in persons with ACS in Indians is not yet studied. Therefore, in the present study, we are evaluating whether WMR can be useful as a marker of prognosis in Indian persons presenting with ACS.

\section{Aims and Objectives}

To detect usefulness of WMR for predicting short-term (30 days) major adverse cardiac events (MACE) in patients presenting with ACS, and also compare values and MACE events in males and females.

\section{Materials and Methods}

The present study was conducted in a tertiary care hospital from September 2020 to December 2020. In the present study, the sample size was 60 cases with ACS, which is based on the study by Adam et al, in which the sensitivity of WMR was $69 \%$ with mortality rate of $14 \%$, and the minimum sample size required was 25 cases with ACS. ${ }^{9}$ Inclusion criteria include patients with ACS. ACS patients were identified by using the following criteria: non-ST-segment elevated myocardial infarction (NSTEMI) was diagnosed if persons had increased cardiac enzymes without detectable ST-segment elevation on the electrocardiogram (ECG). STEMI was diagnosed if person complained of typical angina which lasted for more than 20 minutes along with any one of the following features: ST-segment elevation of at least $1 \mathrm{~mm}$ in two or more contiguous leads, formation of new $\mathrm{Q}$ wave or left bundle branch block (LBBB) formation, and/or two times increase in the cardiac enzymes. Unstable angina (UA) is diagnosed if there is detectable ischemic changes on an ECG without cardiac enzymes elevation. ${ }^{10}$

A total of 60 patients presenting with ACS, who fulfilled the selection criterion, were studied. The clearance from the Institutional Ethical committee (IEC) was obtained prior to the initiation of the study. Eligible patients for the study were explained about the study and investigations to be conducted, and written informed consent was taken. All the necessary data including age and gender, clinical features, history of diabetes mellitus, hypertension, smoking, alcohol consumption, and tobacco chewing were taken from the eligible patients, and physical examination carried out to assess the vital parameters. Patients were also evaluated for Killip clinical examination classification and New York Heart Association (NYHA) classification. ${ }^{11,12}$

\section{Laboratory Investigations:}

After admission of the patient, blood samples were collected within 30 minutes to measure hematological parameters and conduct biochemical investigations.

An automated hematology analyzer Beckmann Coulter DXH800 was used to measure hematological parameters and liver function tests (LFTs), while serum electrolytes, blood urea nitrogen (BUN) and creatinine $(\mathrm{Cr}$ ) were measured with Roche Cobas c501 chemistry analyzer. Patients were also evaluated with ischemia markers creatine phosphokinase (CPK), lactate dehydrogenase (LDH), echocardiography, and a 12-lead ECG. Troponin I was measured with Beckmann DXI600.

\section{Electrocardiographic and Enzymatic Analysis:}

The eligible patients were subjected to ECG in order to diagnose the type of ACS. A standard 12-lead ECG recorded at a paper speed of $25 \mathrm{~mm} / \mathrm{sec}$ and at a calibration of $1 \mathrm{mV}=10 \mathrm{~mm}$. STEMI was diagnosed, according to the European Society of Cardiology (ESC) and American College of Cardiology (ACC) criteria, as typical angina lasting longer than 30 minutes, increased creatine kinase-MB (CK-MB) fraction $>200 \mathrm{U} / \mathrm{l}$ and/or increased cardiac troponin I more than 2 microgram $\mid \mathrm{L}$ and/ or new ST elevation at the J point in two contiguous leads $>0.2 \mathrm{mV}$ which leads v2-v3 or $>0.1 \mathrm{mV}$ in other leads. ${ }^{13,14}$

Echocardiography: The patients were evaluated with echocardiography at the time of hospital admission. The left ventricular end-diastolic diameter was measured from the parasternal long axis view, and the left ventricular ejection fraction was measured using the single-plane, Simpson method.

\section{Inclusion Criteria}

- All patients with clinical suspicion of ACS are included.

- 18 to 90 years age people are included.

- Both gender are included.

\section{Exclusion Criteria}

- Non-ACS patients, patients with malignancy history, inflammatory diseases, autoimmune disorders and infections, and those who are immunosuppressed.

\section{Statistical Analysis}

- Data has been collected, compiled using Microsoft Excel, and statistical analyzed with the help of SSPS v20. Results on continuous variables are presented as mean and standard deviation (SD). Results on categorical variables are presented as percentages. Chi-square test is used to find out the significance of study parameters on a categorical scale between two investigations. Receiver operating characteristic (ROC) curve analysis was conducted to determine prognostic accuracy of WMR in predicting MACE. Continuous variables are analyzed using independent t-tests for normal distribution; otherwise, the Mann-Whitney U-test is employed. All p-values are two-tailed, and $p<0.05$ is considered statistically significant. 


\section{Results}

In this study, a total of 60 patients with ACS were enrolled.

We observed a slightly greater number of males $(n=36$, $60 \%)$ than females $(n=24,40 \%)$ in the present study, as shown in - Table 1.

Comorbidities of patients: Among 60, 36 (60\%) had hypertension and 24 did not have hypertension, 21 (35\%) were diabetic, 26 (43.3\%) were alcoholics, 21(35\%) were smokers, and 10 (16.67\%) had hypothyroid, as shown in - Table 2.

Diagnosis of patients: Out of 60 patients presenting with ACS, 9 had anterior wall myocardial infarction (AWMI), 8 presented with extensive anterior wall myocardial infarction (EAWMI), 4 presented with inferior wall myocardial infarction (IWMI), 1 with IWMI with complete heart block (CHB), 2 had EAWMI, 5 had inferior wall posterior wall myocardial infarction (IWPWMI), 1 had extensive inferior wall posterior wall myocardial infarction (EIWPWMI), 16 had NSTEMI, and 13 presented with UA, as shown in - Table 3.

Out of 60 patients, 24 had single vessel disease (left anterior descending [LAD] 13, left circumflex [LCX] 2, right

Table 2 Comorbidities of patients

\begin{tabular}{|l|l|l|}
\hline Comorbidities & Frequency & Percent \\
\hline Hypertension & 36 & $60 \%$ \\
\hline Diabetes & 21 & $35 \%$ \\
\hline Alcoholism & 26 & $43.3 \%$ \\
\hline Smoking & 21 & $35 \%$ \\
\hline Hypothyroidism & 10 & $16.6 \%$ \\
\hline
\end{tabular}

Table 1 Gender distribution

\begin{tabular}{|l|l|l|}
\hline Gender & Frequency & Percent \\
\hline Female & 24 & $40.00 \%$ \\
\hline Male & 36 & $60.00 \%$ \\
\hline Total & 60 & $100.00 \%$ \\
\hline
\end{tabular}

Abbreviations: AUC, area under curve; MACE, major adverse cardiac event; WMR, white blood cell count to mean platelet volume ratio. coronary artery [RCA] 9), 1 had left main, triple vessel disease, 9 had triple vessel disease, 26 had double vessel disease (LAD LCX 14, LAD RCA 6, LCX RCA 6) disease. Out of 60 patients 15 patients underwent $\mathrm{PCI}$ to LAD, 10 patients underwent PCI to RCA, 4 underwent PCI to LCX, 8 underwent coronary artery bypass graft (CABG) surgery, and 1 underwent multivessel PCI.

In present study, the ROC curve (as shown in - Fig. 1 below) showed cutoff value of WMR as 1059 with area under curve (AUC) of 0.6 (95\% CI 0.4-0.9). The area under ROC curve is maximum at 1059 value with better sensitivity.

MACE was noted in 12 patients and mortality was noted in 6 patients. MACE like recurrent myocardial infarction (REMI) occurred in 6 patients with high WMR (> 1059) and

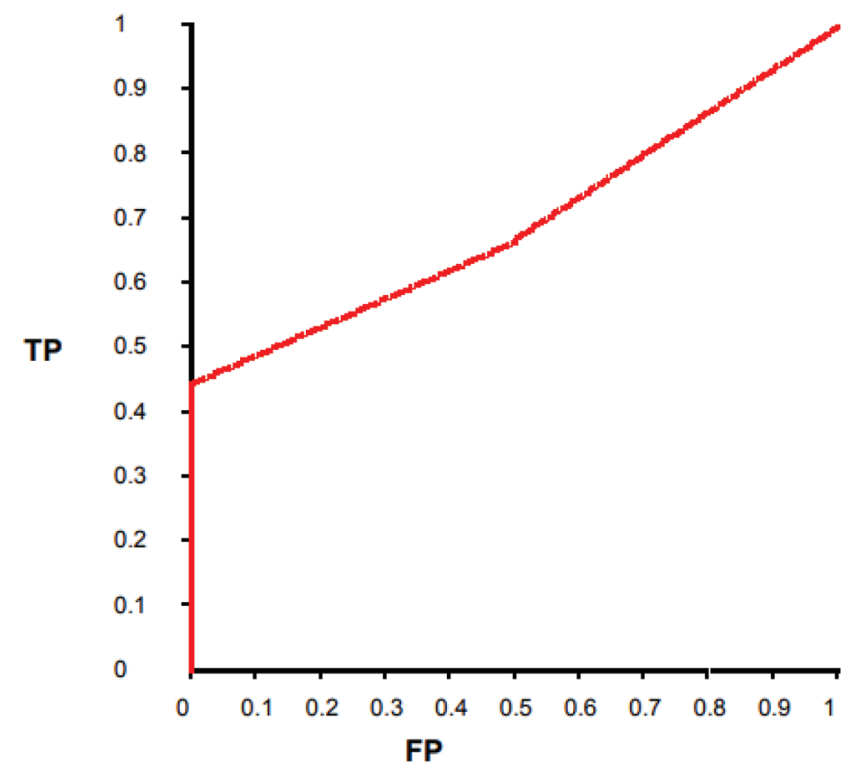

TP - True positive rate, FP - False positive rate

Area under the ROC curve $=0.6(0.4-0.9)$

Fig. 1 Receiver operating characteristic (ROC) curve showing area under curve (AUC) for white blood cell count to mean platelet volume ratio (WMR) in predicting major adverse cardiac event (MACE).

Table 3 Diagnosis of patients

\begin{tabular}{|l|l|l|l|l|l|l|}
\hline Diagnosis & Males & Percentage & Females & Percentage & Total & Percent \\
\hline AWMI & 7 & $11.66 \%$ & 2 & $3.33 \%$ & 9 & $15.00 \%$ \\
\hline EAWMI & 5 & $8.33 \%$ & 3 & $5.00 \%$ & 8 & $13.33 \%$ \\
\hline EIWMI & 0 & $0.00 \%$ & 2 & $3.33 \%$ & 2 & $3.33 \%$ \\
\hline EIWPWMI & 1 & $1.66 \%$ & 0 & $0.00 \%$ & 1 & $1.67 \%$ \\
\hline IWMI & 2 & $3.33 \%$ & 2 & $3.33 \%$ & 4 & $6.67 \%$ \\
\hline IWMI CHB & 1 & $1.66 \%$ & 0 & $0.00 \%$ & 1 & $1.67 \%$ \\
\hline IWPWMI & 3 & $5.00 \%$ & 2 & $3.33 \%$ & 5 & $8.33 \%$ \\
\hline NSTEMI & 9 & $15 \%$ & 7 & $11.66 \%$ & 16 & $26.67 \%$ \\
\hline UA & 7 & $11.66 \%$ & 6 & $10.00 \%$ & 13 & $21.67 \%$ \\
\hline Total & 36 & $60.00 \%$ & 24 & $40.00 \%$ & 60 & $100.00 \%$ \\
\hline
\end{tabular}

Abbreviations: AWMI, anterior wall myocardial infarction; EAWMI, external anterior wall myocardial infarction; EIWMI, external inferior wall myocardial infarction; EIWPWMI, external inferior wall posterior wall myocardial infarction; IWMI, inferior wall myocardial infarction; IWMI CHB, inferior wall myocardial infarction complete heart block; IWPWMI, inferior wall posterior wall myocardial infarction; non-ST-segment elevation myocardial infarction; UA, unstable angina. 
in 1 patient with low WMR (<1059), arrhythmias occurred in 4 patients with high WMR (1059) and in 1 patient with low WMR (1059), and cardiogenic shock occurred in 8 patients with high WMR (1059).

WMR with a cutoff value of 1059 was significant and highly accurate in predicting MACE with sensitivity of $91.6 \%$, specificity of $87.5 \%$, positive predictive value (PPV) of $64.7 \%$, negative predictive value (NPV) of $97.6 \%$, and diagnostic accuracy of $88.3 \%$.

\section{Accuracy Of WMR in Predicting MACE (Overall)}

MACE occurred in 12 patients; among them, 11 patients had WMR ratio > 1059 and 1 patient had low WMR ratio < 1059, with sensitivity of $91.6 \%$, specificity of $87.5 \%$, PPV of $64.7 \%$, NPV of $97.6 \%$, and diagnostic accuracy of $88.3 \%$ (- Table 4 ).

\section{Comparison of WMR Ratio in Males and Females}

In the present study, 17 patients had WMR ratio > 1059; among them, 11 were males and 6 were females ( - Table 5 ).

\section{Accuracy of WMR in Predicting MACE in Females}

MACE occurred in 12 patients; among them, 6 patients were females. Among those 6 females, 5 patients had WMR ratio $>1059$ and 1 patient had low WMR ratio < 1059 with sensitivity of $83.3 \%$, specificity of $94.4 \%$, PPV of $83.33 \%$, NPV of $94.44 \%$, and diagnostic accuracy of $91.67 \%$ (-Table 6 ).

\section{Comparing WMR and MACE}

About 20 MACE events occurred; among them, 18 events occurred in patients with WMR ratio $>1059$ and 2 patients had low WMR ratio < 1059, with sensitivity of $90 \%$, specificity of $37.5 \%$, PPV of $41.6 \%$, NPV of $88.24 \%$, and diagnostic accuracy of $55 \%$ (- Table 7 ).

\section{Various Types of Major Cardiac Events:}

MACE like REMI occurred in 6 patients with high WMR (> 1059) and in 1 patient with low WMR (< 1059), arrhythmias occurred in 4 patients with high WMR (1059) and in 1 patient with low WMR (1059), and cardiogenic shock occurred in 8 patients with high WMR (1059) (-Table 8).

\section{Major Adverse Cardiac Events}

Overall, 20 MACE occurred in 12 patients. Out of them, 6 were males (10\%) and 6 were females (10\%). Hence, in the present study, MACE did not show any gender predilection ( and 10 ).

\section{Comparison of Baseline Investigations with WMR (-Table 11)}

\section{Comparison of Baseline Investigations with MACE (-Table 12)}

\section{Mean Values of Study Population (-Table 13)}

\section{Discussion}

WMR is a novel noninvasive marker, which is highly accurate in predicting MACE in patients presenting with ACS. Raised WMR with cutoff value of 1059 with AUC of 0.6 (95\% CI 0.4-0.9), sensitivity of $91.6 \%$, specificity of $87.5 \%$, PPV of $64.7 \%$, NPV of 97.6\%, and diagnostic accuracy of 88.3\% ( $p=0.01)$.

MACE occurred in 12 patients; among them, 6 patients were females. Compared to males, females had less sensitivity

Table 4 MACE and WMR (patients)

\begin{tabular}{|c|c|c|c|c|c|}
\hline \multirow[t]{2}{*}{ WMR } & \multicolumn{3}{|c|}{ MACE } & \multirow[t]{2}{*}{ Total } & \multirow[t]{2}{*}{$p$-Value } \\
\hline & Yes & \multicolumn{2}{|l|}{ No } & & \\
\hline$>1059$ & 11 & \multicolumn{2}{|l|}{6} & 17 & \multirow[t]{3}{*}{0.01 (Significant) } \\
\hline$<1=1059$ & 1 & \multicolumn{2}{|l|}{42} & 43 & \\
\hline Total & 12 & \multicolumn{2}{|l|}{48} & $60(100 \%)$ & \\
\hline Parameter (patients) & & Estimate & Lower-upper 95\% Cls & \multicolumn{2}{|l|}{ Method } \\
\hline \multicolumn{2}{|l|}{ Sensitivity } & $91.6 \%$ & (61.5-99.79) & \multicolumn{2}{|l|}{ Wilson score } \\
\hline \multicolumn{2}{|l|}{ Specificity } & $87.5 \%$ & $(74.72-95.27)^{1}$ & \multicolumn{2}{|l|}{ Wilson score } \\
\hline \multicolumn{2}{|l|}{ PPV } & $64.71 \%$ & $(45.97-79.80)^{1}$ & \multicolumn{2}{|l|}{ Wilson score } \\
\hline \multicolumn{2}{|l|}{ NPV } & $97.6 \%$ & $(86.512-99.64)^{1}$ & \multicolumn{2}{|l|}{ Wilson score } \\
\hline \multicolumn{2}{|l|}{ Diagnostic accuracy } & $88.3 \%$ & $(77.43-95.18)^{1}$ & \multicolumn{2}{|l|}{ Wilson score } \\
\hline \multicolumn{2}{|l|}{ Likelihood ratio of a positive test } & 7.33 & $(3.04-15.8)$ & & \\
\hline \multicolumn{2}{|l|}{ Likelihood ratio of a negative test } & 0.10 & $(0.012-0.62)$ & & \\
\hline
\end{tabular}

Abbreviations: $\mathrm{Cl}$, confidence interval; MACE, major adverse cardiac event; NPV, negative predictive value; PPV, positive predictive value; WMR, white blood cell count to mean platelet volume ratio. 
Table 5 Comparison of WMR ratio in males and females

\begin{tabular}{|l|l|l|l|l|l|l|}
\hline WMR & Males & Percentage & Females & Percentage & Total & Percent \\
\hline$\leq 1059$ & 25 & $41.66 \%$ & 18 & $30 \%$ & 43 & $71.66 \%$ \\
\hline$>1059$ & 11 & $18.33 \%$ & 6 & $10.00 \%$ & 17 & $28.33 \%$ \\
\hline Total & 36 & $60.00 \%$ & 24 & $40.00 \%$ & 60 & $100.00 \%$ \\
\hline
\end{tabular}

Abbreviation: WMR, white blood cell count to mean platelet volume ratio.

Table 6 MACE and WMR (female patients)

\begin{tabular}{|c|c|c|c|c|c|}
\hline \multirow[t]{2}{*}{ WMR } & \multicolumn{2}{|r|}{ MACE } & \multirow{2}{*}{\multicolumn{2}{|c|}{ Total }} & \multirow[t]{2}{*}{$p$-Value } \\
\hline & Yes & No & & & \\
\hline$>1059$ & 5 & 1 & \multicolumn{2}{|l|}{6} & \multirow[t]{3}{*}{0.01 (significant) } \\
\hline$\leq 1059$ & 1 & 17 & \multicolumn{2}{|l|}{18} & \\
\hline Total & 6 & 18 & \multicolumn{2}{|l|}{24} & \\
\hline Parameter (patients) & & Estimate & Lower-upper 95\% Cls & Method & \\
\hline \multicolumn{2}{|l|}{ Sensitivity } & $83.3 \%$ & $(35.88-99.58)$ & \multicolumn{2}{|l|}{ Wilson score } \\
\hline \multicolumn{2}{|l|}{ Specificity } & $94.4 \%$ & $\left(72.71-99.86^{1}\right)$ & \multicolumn{2}{|l|}{ Wilson score } \\
\hline \multicolumn{2}{|l|}{ PPV } & $83.33 \%$ & $\left(41.86-97.20^{1}\right)$ & \multicolumn{2}{|l|}{ Wilson score } \\
\hline \multicolumn{2}{|l|}{ NPV } & $94.44 \%$ & $\left(73.89-99.03^{1}\right)$ & \multicolumn{2}{|l|}{ Wilson score } \\
\hline \multicolumn{2}{|l|}{ Diagnostic Accuracy } & $91.67 \%$ & $\left(73.00-98.97^{1}\right)$ & \multicolumn{2}{|l|}{ Wilson score } \\
\hline \multicolumn{2}{|l|}{ Likelihood ratio of a positive test } & 15 & $(2.162-104.18)$ & & \\
\hline \multicolumn{2}{|l|}{ Likelihood ratio of a negative test } & 0.18 & $(0.03-1.06)$ & & \\
\hline
\end{tabular}

Abbreviations: $\mathrm{Cl}$, confidence interval; MACE, major adverse cardiac event; NPV, negative predictive value; PPV, positive predictive value; WMR, white blood cell count to mean platelet volume ratio.

Table 7 MACE and WMR: (events)

\begin{tabular}{|c|c|c|c|c|c|}
\hline \multirow[t]{2}{*}{ WMR } & \multicolumn{2}{|c|}{ MACE } & \multirow{2}{*}{\multicolumn{2}{|c|}{ Total }} & \multirow[t]{2}{*}{$p$-Value } \\
\hline & Yes & No & & & \\
\hline$>1059$ & $18(30 \%)$ & $25(41.66 \%)$ & \multicolumn{2}{|l|}{$43(71.66 \%)$} & \multirow{3}{*}{$\begin{array}{l}0.01 \\
\text { (significant) }\end{array}$} \\
\hline$\leq 1059$ & $2(3.33 \%)$ & $15(25 \%)$ & \multicolumn{2}{|l|}{$17(28.33 \%)$} & \\
\hline Total & $20(33.33 \%)$ & $40(66.66 \%)$ & \multicolumn{2}{|l|}{$60(100 \%)$} & \\
\hline Parameter (events) & & Estimate & Lower-upper $95 \%$ Cls & Method & \\
\hline \multicolumn{2}{|l|}{ Sensitivity } & $90 \%$ & $\left(69.9-97.21^{1}\right)$ & \multicolumn{2}{|l|}{ Wilson score } \\
\hline \multicolumn{2}{|l|}{ Specificity } & $37.5 \%$ & $\left(24.22-52.97^{1}\right)$ & \multicolumn{2}{|l|}{ Wilson score } \\
\hline \multicolumn{2}{|l|}{ PPV } & $41.86 \%$ & $\left(28.38-56.67^{1}\right)$ & \multicolumn{2}{|l|}{ Wilson score } \\
\hline \multicolumn{2}{|l|}{ NPV } & $88.24 \%$ & $\left(65.66-96.71^{1}\right)$ & \multicolumn{2}{|l|}{ Wilson score } \\
\hline \multicolumn{2}{|l|}{ Diagnostic accuracy } & $55 \%$ & $\left(42.49-66.91^{1}\right)$ & \multicolumn{2}{|l|}{ Wilson score } \\
\hline \multicolumn{2}{|c|}{ Likelihood ratio of a positive test } & 1.44 & $(1.315-1.576)$ & & \\
\hline \multicolumn{2}{|c|}{ Likelihood ratio of a negative test } & 0.2667 & $(0.0805-0.8834)$ & & \\
\hline \multicolumn{2}{|l|}{ Diagnostic odds } & 5.4 & $(1.096-26.61)$ & & \\
\hline
\end{tabular}

Abbreviations: $\mathrm{Cl}$, confidence interval; MACE, major adverse cardiac event; NPV, negative predictive value; PPV, positive predictive value; WMR, white blood cell count to mean platelet volume ratio. 
Table 8 Various types of major cardiac events with high and low WMR

\begin{tabular}{|l|l|l|l|}
\hline \multirow{2}{*}{ MACE } & \multicolumn{2}{|c|}{ WMR } & \multirow{2}{*}{ Total } \\
\cline { 2 - 3 } & $\geq 1059$ & $<1059$ & \\
\hline REMI & $6(30 \%)$ & $1(5 \%)$ & $7(35 \%)$ \\
\hline Arrythmia & $4(20 \%)$ & $1(5 \%)$ & $5(25 \%)$ \\
\hline Cardiogenic Shock & $8(40 \%)$ & 0 & $8(40 \%)$ \\
\hline Total & $18(90 \%)$ & $2(10 \%)$ & $20(100 \%)$ \\
\hline
\end{tabular}

Abbreviations: MACE, major adverse cardiac event; REMI, recurrent myocardial infarction; WMR, white blood cell count to mean platelet volume ratio.

Table 9 MACE

\begin{tabular}{|l|l|l|}
\hline MACE & No. of events & Percent \\
\hline Yes & 20 & $33.33 \%$ \\
\hline No & 40 & $66.66 \%$ \\
\hline Total & 60 & $100.00 \%$ \\
\hline
\end{tabular}

Abbreviation: MACE, major adverse cardiac event.

Table 10 Comparison of MACE in males and females

\begin{tabular}{|l|l|l|l|l|l|l|}
\hline MACE & Males & Percentage & Females & Percentage & Total & Percent \\
\hline Yes & 6 & $10 \%$ & 6 & $10 \%$ & 12 & $20.00 \%$ \\
\hline No & 30 & $50.00 \%$ & 18 & $30.00 \%$ & 48 & $80.00 \%$ \\
\hline Total & 36 & $60.00 \%$ & 24 & $40.00 \%$ & 60 & $100.00 \%$ \\
\hline
\end{tabular}

Abbreviation: MACE, major adverse cardiac event.

Table 11 Comparison of baseline investigations with WMR

\begin{tabular}{|c|c|c|c|c|}
\hline \multicolumn{2}{|l|}{ Parameter } & \multirow{2}{*}{$\begin{array}{l}\text { WMR }(<1059)(n=43) \\
58 \pm 10.399\end{array}$} & \multirow{2}{*}{$\begin{array}{l}\text { WMR }(>1059)(n=17) \\
55.2 \pm 11.71\end{array}$} & \multirow{2}{*}{$\begin{array}{l}p \text {-Value } \\
0.3\end{array}$} \\
\hline & Age & & & \\
\hline \multirow{8}{*}{$\begin{array}{l}\text { Complete blood } \\
\text { picture }\end{array}$} & Hemoglobin & $11.72 \pm 1.27$ & $12.511 \pm 1.30$ & $0.03^{*}$ \\
\hline & WBC & $9165.116 \pm 1557.299$ & $14413.529 \pm 1795.79$ & $<0.0000001^{*}$ \\
\hline & PCV & $39.72 \pm 5.006$ & $42.117 \pm 5.218$ & 0.1 \\
\hline & PLT & $1.98 \pm 0.407$ & $2.5 \pm 0.306$ & $0.00001^{*}$ \\
\hline & Neutrophils & $7.19 \pm 1.55$ & $11.37 \pm 1.796$ & $<0.0000001^{*}$ \\
\hline & Lymphocytes & $1.62 \pm 0.370$ & $2.02 \pm 0.405$ & $0.0005^{*}$ \\
\hline & MPV & $10.82 \pm 1.239$ & $11.78 \pm 1.449$ & $0.01^{*}$ \\
\hline & WMR & $840.34 \pm 141.83$ & $11247 \pm 127.65$ & $<0.000001^{*}$ \\
\hline \multirow[t]{2}{*}{ Cardiac enzymes } & CPK-MB & $259.62 \pm 128.55$ & $434.823 \pm 174.86$ & $0.00007^{*}$ \\
\hline & $\mathrm{LDH}$ & $362.72 \pm 167.95$ & $573.47 \pm 206.95$ & $0.0001^{*}$ \\
\hline \multirow[t]{4}{*}{ Liver enzymes } & AST & $63.62 \pm 98.86$ & $186.529 \pm 317.04$ & $0.02^{*}$ \\
\hline & ALT & $56.34 \pm 75.146$ & $158.647 \pm 242.28$ & $0.01^{*}$ \\
\hline & ALP & $205.34 \pm 68.379$ & $201.88 \pm 71.37$ & 0.8 \\
\hline & Bilirubin & $1.011 \pm 0.3148$ & $1.0176 \pm 0.367$ & 0.9 \\
\hline \multirow[t]{2}{*}{ Renal parameters } & Blood urea & $23.81 \pm 11.11$ & $30.117 \pm 16.78$ & 0.09 \\
\hline & Serum creatinine & $0.934 \pm 0.43$ & $1.417 \pm 1.067$ & $0.01^{*}$ \\
\hline \multirow[t]{5}{*}{ Lipid profile } & Total cholesterol & $170.186 \pm 55.23$ & $202.70 \pm 78.66$ & 0.07 \\
\hline & $\mathrm{LDL}$ & $120.65 \pm 31.65$ & $144.23 \pm 46.866$ & $0.03^{*}$ \\
\hline & $\mathrm{HDL}$ & $38.65 \pm 3.81$ & $36.1176 \pm 4.32$ & $0.02^{*}$ \\
\hline & TG & $234.093 \pm 135.46$ & $352.70 \pm 158.077$ & $0.005^{*}$ \\
\hline & VLDL & $40.27 \pm 14.66$ & $39.05 \pm 13.325$ & 0.7 \\
\hline
\end{tabular}

Abbreviations: ALP, alkaline phosphatase; ALT, alanine transaminase; AST, aspartate aminotransferase; CPK-MB, creatine phosphokinase-MB; HDL, high-density lipoprotein; LDH, lactate dehydrogenase; LDL, low-density lipoprotein; MPV, mean platelet volume; PCV, packed cell volume; PLT, platelet count; TG, triglyceride; VLDL, very low density lipoprotein; WBC, white blood cell; WMR, white blood cell count to mean platelet volume ratio.

*Pertains to statistically significant $(p \leq 0.05)$. 
Table 12 Comparison of baseline investigations with MACE

\begin{tabular}{|c|c|c|c|c|}
\hline \multicolumn{2}{|l|}{ Parameter } & \multirow{2}{*}{$\begin{array}{l}\text { MACE not reported (48) } \\
58.0625 \pm 10.78\end{array}$} & \multirow{2}{*}{$\begin{array}{l}\text { MACE reported (12) } \\
54.33 \pm 10.48\end{array}$} & \multirow{2}{*}{$\begin{array}{l}p \text {-value } \\
0.2\end{array}$} \\
\hline & Age & & & \\
\hline \multirow[t]{8}{*}{ Complete blood picture } & Hemoglobin & $11.745 \pm 1.269$ & $12.74 \pm 1.28$ & $0.01^{*}$ \\
\hline & WBC & $9647.9116 \pm 1906.31$ & $14669.16 \pm 2632.169$ & $<0.0000001^{*}$ \\
\hline & PCV & $40 \pm 4.61$ & $42 \pm 6.87$ & 0.2 \\
\hline & PLT & $2.066 \pm 0.444$ & $2.83 \pm 0.363$ & $0.0000007^{*}$ \\
\hline & Neutrophils & $7.56 \pm 1.77$ & $11.55 \pm 2.47$ & $<0.000001^{*}$ \\
\hline & Lymphocytes & $1.63 \pm 0.368$ & $2.19 \pm 0.336$ & $0.00001^{*}$ \\
\hline & MPV & $10.922 \pm 1.22$ & $11.925 \pm 1.611$ & $0.02^{*}$ \\
\hline & WMR & $889.375 \pm 163.96$ & $1220.33 \pm 270.55$ & $0.000001^{*}$ \\
\hline \multirow[t]{2}{*}{ Cardiac enzymes } & CPK-MB & $267.29 \pm 132.70$ & $477.166 \pm 166.98$ & $0.00001^{*}$ \\
\hline & $\mathrm{LDH}$ & $371.04 \pm 171.839$ & $628 \pm 188.171$ & $0.00002^{*}$ \\
\hline \multirow[t]{4}{*}{ Liver enzymes } & AST & $73.75 \pm 182.54$ & $197.25 \pm 211.11$ & $0.04^{*}$ \\
\hline & ALT & $61.39 \pm 119.54$ & $181.08 \pm 212.08$ & $0.01^{*}$ \\
\hline & ALP & $197.125 \pm 63.44$ & $233.33 \pm 83.25$ & 0.1 \\
\hline & Bilirubin & $0.977 \pm 0.269$ & $1.158 \pm 0.48$ & 0.08 \\
\hline \multirow[t]{2}{*}{ Renal parameters } & Blood urea & $23.54 \pm 10.65$ & $38.33 \pm 18.64$ & $0.0005^{*}$ \\
\hline & Serum creatinine & $0.929 \pm 0.408$ & $1.64 \pm 1.21$ & $0.001^{*}$ \\
\hline \multirow[t]{5}{*}{ Lipid profile } & Total cholesterol & $163.58 \pm 49.83$ & $242.666 \pm 75.83$ & $0.00004^{*}$ \\
\hline & $\mathrm{LDL}$ & $117.166 \pm 29.31$ & $168 \pm 41.34$ & $0.000007^{*}$ \\
\hline & $\mathrm{HDL}$ & $38.45 \pm 3.89$ & $35.83 \pm 4.38$ & $0.04^{*}$ \\
\hline & TGS & $227.208 \pm 125.56$ & $429.66 \pm 137.30$ & $0.000007^{*}$ \\
\hline & VLDL & $39.833 \pm 14.509$ & $40.33 \pm 13.47$ & 0.9 \\
\hline
\end{tabular}

Abbreviations: ALP, alkaline phosphatase; ALT, alanine transaminase; AST, aspartate aminotransferase; CPK-MB, creatine phosphokinase-MB; HDL, high-density lipoprotein; LDH, lactate dehydrogenase; LDL, low-density lipoprotein; MPV, mean platelet volume; PCV, packed cell volume; PLT, platelet count; TG, triglyceride; VLDL, very low density lipoprotein; WBC, white blood cell; WMR, white blood cell count to mean platelet volume ratio.

*Pertains to Statistically Significan $(p \leq 0.05)$.

Table 13 Mean values of study population

\begin{tabular}{|c|c|c|}
\hline \multicolumn{2}{|l|}{ Parameter } & \multirow{2}{*}{$\begin{array}{l}\text { Mean } \pm \text { SD } \\
11.945 \pm 1.32\end{array}$} \\
\hline Complete blood picture & Hemoglobin & \\
\hline & WBC & $10652.166 \pm 28779.07$ \\
\hline & PCV & $40.4 \pm 5.139$ \\
\hline & PLT & $2.13 \pm 0.44$ \\
\hline & Neutrophils & $8.36 \pm 2.49$ \\
\hline & Lymphocytes & $1.73 \pm 0.42$ \\
\hline & MPV & $11.123 \pm 1.355$ \\
\hline & WMR & $955.56 \pm 229.97$ \\
\hline \multirow[t]{2}{*}{ Cardiac enzymes } & CPK-MB & $309.266 \pm 162.46$ \\
\hline & $\mathrm{LDH}$ & $422.43 \pm 202.158$ \\
\hline \multirow[t]{4}{*}{ Liver enzymes } & AST & $98.45 \pm 193.22$ \\
\hline & ALT & $85.33 \pm 148.66$ \\
\hline & ALP & $204.366 \pm 68.64$ \\
\hline & Bilirubin & $1.01 \pm 0.32$ \\
\hline \multirow[t]{2}{*}{ Renal parameters } & Blood urea & $25.6 \pm 13.13$ \\
\hline & Serum creatinine & $1.071 \pm 0.699$ \\
\hline \multirow[t]{5}{*}{ Lipid profile } & Total cholesterol & $179.4 \pm 63.78$ \\
\hline & $\mathrm{LDL}$ & $127.3 \pm 37.73$ \\
\hline & $\mathrm{HDL}$ & $37.93 \pm 4.09$ \\
\hline & TGS & $267.7 \pm 150.8$ \\
\hline & VLDL & $39.9 \pm 14.19$ \\
\hline
\end{tabular}

Abbreviations: ALP, alkaline phosphatase; ALT, alanine transaminase; AST, aspartate aminotransferase; CPK-MB, creatine phosphokinase-MB; HDL, high-density lipoprotein; LDH, lactate dehydrogenase; LDL, low-density lipoprotein; MPV, mean platelet volume; PCV, packed cell volume; PLT, platelet count; SD, standard deviation; TG, triglyceride; VLDL, very low density lipoprotein; WBC, white blood cell; WMR, white blood cell count to mean platelet volume ratio. 
to, high specificity to, and high diagnostic accuracy of WMR ratio in predicting MACE.

Overall, 20 MACE occurred in 12 patients. Out of them, 6 were males (10\%) and 6 were females (10\%). Hence, in the present study, MACE did not show any gender predilection.

Out of 60 patients presenting with ACS, 7 patients developed REMI (11.66\%). Among them, 3 were males (5\%) and 4 were females (6.66\%). Compared to males, females had slight increase of incidence of REMI.

Out of 60 patients presenting with ACS, 5 patients developed arrhythmias (8.33\%). Among them, 3 were males (5\%) and 2 were females (3.3\%). Compared to males, females had slight decrease of incidence of arrhythmias.

Out of 60 patients 8 patients developed cardiogenic shock. Among them, 4 were males $(6.66 \%)$ and 4 were females (6.66\%). Compared to males, females had equal incidence of cardiogenic shock.

Out of 60 patients, death occurred in 6 patients in the present study. Among them, 3 were males (5\%) and 3 were females (5\%). Males and females had similar death rates.

Out of 60 patients 6 patients died, and cause of death was cardiogenic shock in 5 and arrhythmia in 1 patient. Cause of death in males was cardiogenic shock, and in females, cause of death was cardiogenic shock and arrhythmia.

Along with WMR, higher WBC and higher neutrophil count were other predicators of MACE.

WMR is a ratio derived from WBC count to MPV ratio. In the present study, baseline MPV level in patients with MACE was $11.78 \pm 1.449$, and in patients without MACE, it was $10.82 \pm 1.239$.

In the present study, mean WBC count was found significantly high in patients with MACE (14669.16 \pm 2632.169$)$ than those without MACE $(9647.9116 \pm 1906.31)$ and the difference was significant $(p<0.0001)$. An increased WBC count is a prominent indicator of compromised microvascular reperfusion. Barron et al presented that acute MI patients with a leucocyte count in the highest quintile had a higher 30-day mortality rate than patients with a leucocyte count in the lower quintiles. ${ }^{15}$ In the present study, it was also found that increased WBC count was associated with MACE.

The present study demonstrated that raised WMR, that is with a cutoff value of 1059 , holds greater predictive value for short-term MACE in patients presenting with ACS, yielding higher diagnostic accuracy (88.3\%), sensitivity (91\%), specificity (87.5\%) with low PPV (64.7\%) and high NPV (97.6\%) as well as high positive likelihood ratio (7.33) and lower negative likelihood ratio $(0.10)(p=0.01)$. Recently, Adam et al reported sensitivity of $68.3 \%$ and specificity of $63.7 \%$ with slightly higher WMR cutoff values (1068.75, AUC $=0.73495 \%$ CI: $0.656-0.812 ; p<0.001$ ), which is very much comparable with the present study.

The present study results show that WMR had a higher sensitivity to predict short-term outcomes; therefore, calculating WMR is not only sensitive but a faster and a more efficient marker.
Overall, the present study demonstrates that raised WMR ( $\geq 1059)$ is highly accurate in discriminating poor outcome, that is, MACE within 30 days of patients presenting with ACS.

However, these conclusions require careful interpretation due to potential limitations of this study

\section{Limitations of this Study}

The conclusions drawn from the present study were based on a single-centre study comprising a relatively smaller sample size, using a nonrandomized sampling technique. Furthermore, we did not measure other more specific proinflammatory markers, including P-selectin, high-sensitivity C-reactive protein (CRP), interleukins, selectin molecules, adhesion ligands and receptors, and markers of oxidative stress to show any comparison between WMR and such biomarkers. Further studies are required to validate these results and define the exact role of WMR in predicting short-term MACE.

\section{Conclusion}

In patients who presented with ACS, high WMR values were associated with worse short-term outcomes and independently predicted short-term MACE. Compared to males, females had less sensitivity, high specificity, and high diagnostic accuracy of WMR ratio in predicting MACE.

\section{Audio}

Audio file for this article is available at https://doi. org/10.1055/s-0041-1732513.

\section{Conflict of Interest}

None declared.

\section{References}

1 Murray CJ, Lopez AD. Mortality by cause for eight regions of the world: Global Burden of Disease Study. Lancet 1997;349(9061): 1269-1276

2 Gupta R, Joshi P, Mohan V, Reddy KS, Yusuf S. Epidemiology and causation of coronary heart disease and stroke in India. Heart 2008;94(1):16-26

3 Libby P. Mechanisms of acute coronary syndromes and their implications for therapy. NEnglJMed 2013;368(21):2004-2013

4 Margolis KL, Manson JE, Greenland P, et al. Women's Health Initiative Research Group. Leukocyte count as a predictor of cardiovascular events and mortality in postmenopausal women: the Women's Health Initiative Observational Study. Arch Intern Med 2005;165(5):500-508

5 Rognoni A, Cavallino C, Veia A, et al. Pathophysiology of atherosclerotic plaque development. Cardiovasc Hematol Agents Med Chem 2015;13(1):10-13 
6 Horne BD, Anderson JL, John JM, et al. Intermountain Heart Collaborative Study Group. Which white blood cell subtypes predict increased cardiovascular risk? J Am Coll Cardiol 2005;45(10):1638-1643

7 Davì G, Patrono C. Platelet activation and atherothrombosis. N Engl J Med 2007;357(24):2482-2494

8 Dehghani MR, Rezaei Y, Fakour S, Arjmand N. White blood cell count to mean platelet volume ratio is a prognostic factor in patients with non-ST elevation acute coronary syndrome with or without metabolic syndrome. Korean Circ J 2016;46(2):229-238

9 Adam AM, Rizvi AH, Haq A, et al. Prognostic value of blood count parameters in patients with acute coronary syndrome. Indian Heart J 2018;70(2):233-240

10 Dehghani MR, Rezaei Y, Taghipour-Sani L. White blood cell count to mean platelet volume ratio as a novel non-invasive marker predicting long-term outcomes in patients with non-ST elevation acute coronary syndrome. Cardiol J 2015;22(4):437-445
11 Mello BH, Oliveira GB, Ramos RF, et al. Validation of the Killip-Kimball classification and late mortality after acute myocardial infarction. Arq Bras Cardiol 2014;103(2):107-117

12 Raphael C, Briscoe C, Davies J, et al. Limitations of the New York Heart Association functional classification system and self-reported walking distances in chronic heart failure. Heart 2007;93(4):476-482

13 Thygesen K, Alpert JS, White HD; Joint ESC/ACCF/AHA/WHF Task Force for the Redefinition of Myocardial Infarction. Universal definition of myocardial infarction. Eur Heart J 2007;28(20):2525-2538

14 Jameson JL, Fauci AS, Kasper DL, Hauser SL, Longo DL, Loscalzo J, Harrison's Principles of Internal Medicine, New York, NY: McGraw-Hill Companies; 2015

15 Barron HV, Harr SD, Radford MJ, Wang Y, Krumholz HM. The association between white blood cell count and acute myocardial infarction mortality in patients $>$ or $=65$ years of age: findings from the cooperative cardiovascular project. J Am Coll Cardiol 2001;38(6):1654-1661 